\title{
First genetic linkage map of Lathyrus cicera based on RNA sequencing-derived markers: Key tool for genetic mapping of disease resistance
}

\author{
Carmen Santos (1)', Nuno Felipe Almeida (10', Mara Lisa Alves ${ }^{1}$, Ralf Horres², Nicolas Krezdorn², Susana Trindade Leitão ${ }^{1}$, \\ Thaïs Aznar-Fernández ${ }^{3}$, Björn Rotter ${ }^{2}$, Peter Winter ${ }^{2}$, Diego Rubiales ${ }^{3}$ and Maria Carlota Vaz Patto ${ }^{1}$
}

\begin{abstract}
The Lathyrus cicera transcriptome was analysed in response to rust (Uromyces pisi) infection to develop novel molecular breeding tools with potential for genetic mapping of resistance in this robust orphan legume species. One RNA-seq library each was generated from control and rust-inoculated leaves from two L. cicera genotypes with contrasting quantitative resistance, de novo assembled into contigs and sequence polymorphisms were identified. In toto, 19,224 SNPs differentiate the susceptible from the partially resistant genotype's transcriptome. In addition, we developed and tested 341 expressed E-SSR markers from the contigs, of which 60.7\% varied between the two L. cicera genotypes. A first L. cicera linkage map was created using part of the developed markers in a RIL population from the cross of the two genotypes. This map contains 307 markers, covered $724.2 \mathrm{cM}$ and is organised in 7 major and 2 minor linkage groups, with an average mapping interval of $2.4 \mathrm{cM}$. The genic markers also enabled us to compare their position in $L$. cicera map with the physical position of the same markers mapped on Medicago truncatula genome, highlighting a high macrosyntenic conservation between both species. This study provides a large new set of genic polymorphic molecular markers with potential for mapping rust resistances. It represents the first step towards genomics-assisted precision breeding in L. cicera.
\end{abstract}

\section{Introduction}

Lathyrus cicera L., the chickling pea, is an annual legume belonging to the tribe Fabeae ${ }^{1,2}$. It is mainly grown as feedstock, both as fodder and grain ${ }^{3}$. However, as far as human consumption is concerned, L. cicera is eaten uncooked as green snack $^{4}$. L. cicera adapts well to harsh environments. It is resistant to drought, water$\operatorname{logging}^{5}$ and to several important legume pathogens. Sources for resistance to rust ${ }^{6}$, powdery mildew ${ }^{7}$, bacterial

Correspondence: Maria Carlota Vaz Patto (cpatto@itqb.unl.pt) ${ }^{1}$ Instituto de Tecnologia Química e Biológica António Xavier, Universidade Nova de Lisboa, Av. da República, Oeiras 2780-157, Portugal

${ }^{2}$ GenXPro GmbH, Frankfurt am Main D-60438, Germany

Full list of author information is available at the end of the article.

These authors contributed equally: Carmen Santos and Nuno Felipe Almeida blight $^{8}$ and crenate broomrape ${ }^{9}$ have been identified. Thus, $L$. cicera is a good option for cropping systems in marginal lands and can function also as a source of resistance genes for transfer to related species such as pea $^{10}$.

A linkage map of $L$. cicera will be crucial to identify and locate the genes and genomic regions responsible for the resistance traits, paving the way to marker assisted selection (MAS) in this orphan species. Indeed, limited genomic resources exist for this plant species, hampering its potential exploitation in legume breeding. In January 2018, the NCBI database contained only 126 sequences (considering DNA and RNA data) with almost no relevance for breeding. However, molecular tools from related species were useful in this legume. Actually, 138

\section{(c) The Author(s) 2018}

(c) (i) Open Access This article is licensed under a Creative Commons Attribution 4.0 International License, which permits use, sharing, adaptation, distribution and reproduction in any medium or format, as long as you give appropriate credit to the original author(s) and the source, provide a link to the Creative Commons license, and indicate if changes were made. The images or other third party material in this article are included in the article's Creative Commons license, unless indicated otherwise in a credit line to the material. If material is not included in the article's Creative Commons license and your intended use is not permitted by statutory regulation or exceeds the permitted use, you will need to obtain permission directly from the copyright holder. To view a copy of this license, visit http://creativecommons.org/licenses/by/4.0/. 
molecular markers of different types previously developed for other legume species, were successfully crossamplified in $L$. cicera $^{11}$.

Rust fungi and particularly those from the genus Uromyces cause important legume diseases. Uromyces pisi (Pers.) Wint. causes pea rust ${ }^{12}$ and is also capable of infecting other species in the genera Lathyrus ${ }^{6,13}$, Vicia and Lens ${ }^{14,15}$.

Lathyrus sativus is the species most closely related to $L$. cicera and may even be derived from $\mathrm{it}^{16}$. These two species might, therefore, share many physiological/genetic stress-response mechanisms. We have recently characterised in more detail the response of L. sativus transcriptome to inoculation with $U$. pisi ${ }^{17}$. Differences in the reaction to infection between the resistant and susceptible genotypes investigated appeared to be mainly due to the activation of the salicylic acid (SA) pathway and expression of several pathogenesis-related (PR) genes. Indeed, in previous studies both species, L. sativus and L. cicera, showed a compatible reaction to $U$. pisi. Also, similar defence mechanisms were present, although $L$. sativus genotypes were more resistant than the $L$. cicera genotypes investigated ${ }^{6,13}$.

In the present work, we investigate the transcriptomes of control and rust-inoculated leaves from two L. cicera genotypes, with contrasting quantitative resistance to rust, to achieve several aims, such as the following: (i) to develop novel expressed simple sequence repeat (E-SSR) and single-nucleotide polymorphism (SNP)-based molecular markers for future mapping and diversity studies in L. cicera, (ii) to examine the differential expression of allelic variants in the two contrasting genotypes after rust inoculation and, based on the SNP information within candidate alleles, (iii) to develop appropriate assays for future quantitative trait loci (QTL)/expression QTL analysis for rust resistance in L. cicera. Finally, we used the developed $L$. cicera molecular markers to construct the first $L$. cicera linkage map and perform a comparative/ synteny study of $L$. cicera with other legume species. This map was developed using a recombinant inbred line (RIL) population resulting from the cross of the two contrasting L. cicera genotypes previously analysed.

\section{Results}

The RNA-seq transcriptomes of $L$. cicera genotypes with contrasting resistance levels

The RNA-seq library of the susceptible genotype BGE008277 comprised 18,395,860 sequencing reads, which were assembled into 66,210 contigs, ranging in size from 150 to $8664 \mathrm{bp}$, with a mean contig length of $537 \mathrm{bp}$. The respective library from the partially resistant genotype BGE023542 comprised 30,320,831 reads, which assembled into 64,382 contigs, with a size range of 150 to $9694 \mathrm{bp}$ and a mean contig length of $571 \mathrm{bp}$.
The de novo reference assembly combining the RNAseq reads from both genotypes and from all treatments comprised 145,985 contigs, ranging in size from 150 to $13,916 \mathrm{bp}$, with a mean contig length of $485 \mathrm{bp}$. BUSCO analysis indicated that our assembly is $86 \%$ complete, showing only $4.9 \%$ of fragmented BUSCOs. In more detail, a total of 1440 BUSCO groups were found: 1238 complete BUSCOs (86\%), from which 1206 single-copy BUSCOs (83.8\%) and 32 duplicated BUSCOs (2.2\%); fragmented BUSCOs: 70 (4.9\%) and missing BUSCOs: 132 (9.1\%). The mapping and quantification of both genotypes' libraries to the reference assembly allowed the analysis of their differential expression in response to $U$. pisi infection. A total of 20,362 contigs were unique to the partially resistant and 12,114 contigs were unique to the susceptible genotype. This de novo Transcriptome Shotgun Assembly project has been deposited at DDBJ/EMBL/ GenBank under the accession PRJNA264792.

\section{Differential gene expression in partially resistant and susceptible $L$. cicera genotypes to rust infection}

L. cicera contigs from the susceptible and partially resistant genotype, respectively that were differentially expressed in response to rust inoculation were grouped into eight expression pattern groups $(\mathrm{A}-\mathrm{H})$ based on their up- or down-regulation $\left(\log _{2} \geq 2\right.$ or $\log _{2} \leq-2$; respectively, $q$ value $\leq 0.05$ ), considering only the transcripts present in all libraries. The number of differentially expressed contigs and description of each group is summarised in Table 1. Groups with most abundant contigs were group $\mathrm{F}$ (contigs downregulated in both genotypes) and $\mathrm{H}$ (contigs downregulated only in the partially resistant genotype) with 4520 and 3498 contigs, respectively. They were followed by a group that included 2161 entries upregulated upon infection in both genotypes (group A). A detailed list including all identified contigs, their description and assignment to expression pattern groups can be found in Supplementary Table S1. As depicted in Supplementary Fig. S1, from the 111,287 contigs that could be identified and quantified, 43,590 were shared among all libraries.

\section{Annotation of $L$. cicera contigs}

From the 111,287 contigs detected in all libraries, 46,588 (41.9\%) were matched via BLAST to entries in plant databases. A total of $622(0.6 \%)$ contigs matched only to fungal databases and were present only in the inoculated libraries. In addition to these, $688(0.6 \%)$ other contigs, that were also absent in non-inoculated samples, had a higher hit-score in fungal databases than in plant databases. Thus, a total of $1.2 \%$ of all contigs most probably correspond to $U$. pisi sequences.

As indicated in Fig. 1, BLAST produced hits mainly to other legume species. Medicago truncatula (23,754; 
Table 1 Classification of contigs according to their differential expression in the susceptible and resistant genotype upon infection with $U$. pisi

\begin{tabular}{lll}
$\begin{array}{l}\text { Expression } \\
\text { pattern group }\end{array}$ & Feature & $\begin{array}{l}\text { \# of } \\
\text { contigs }\end{array}$ \\
\hline A & $\begin{array}{l}\text { Upregulated in Resistant } \\
\text { Upregulated in Susceptible }\end{array}$ & 2,161 \\
B & $\begin{array}{l}\text { Upregulated in Resistant } \\
\text { Upregulated in Susceptible, higher in }\end{array}$ & 12 \\
& Susceptible \\
C & Upregulated in Resistant, higher in Resistant & 20 \\
& Upregulated in Susceptible & \\
D & Upregulated in Susceptible & 1,715 \\
E & Upregulated in Resistant & 338 \\
F & Downregulated in Resistant & 4,520 \\
G & Downregulated in Susceptible & \\
H & Downregulated in Susceptible & 1,399 \\
Total & Downregulated in Resistant & 3,498 \\
\hline
\end{tabular}

Upregulated: $\left(\log _{2} \geq 2 ; q\right.$ value $\left.\leq 0.05\right)$; Downregulated: $\left(\log _{2} \leq-2 ; q\right.$ value $\left.\leq 0.05\right)$; higher in Susceptible: $\left(\log _{2}\right.$ fold change between all resistant and susceptible genotype contigs $\leq-2$; $q$ value $\leq 0.05$ ); higher in Resistant: ( $\log _{2}$ fold change between all resistant and susceptible genotype contigs $\geq 2 ; q$ value $\leq 0.05$ ).
50.99\%), Cicer arietinum (11,177; 23.99\%), Glycine max (3,559; 7.64\%), Pisum sativum (1,224; 2.63\%), Phaseolus vulgaris (800; $1.72 \%$ ) and Lotus japonicus (300; 0.64\%) were the best matching legume species. Vitis vinifera (1,128; 2.42\%), Hordeum vulgare (307; 0.66\%), Zea mays (216; 0.46\%) and the model Arabidopsis thaliana (214; $0.46 \%)$ were the best matching non-legume species.

As depicted in Fig. 2, functional annotation of all differentially expressed plant contigs via Mercator and MapMan, grouped them into 34 main functional categories, of which the categories 'protein' (11.5\%), 'RNA' (8.7\%), 'signalling' (6.4\%), 'transport' (5.2\%), 'miscellaneous' (4.7\%), and 'stress' (3.9\%) were most represented. A total of $37.4 \%$ differentially expressed (DE) contigs could not be assigned to any functional category.

The analysis of the identified functional categories revealed transcripts that are potentially involved in several layers of defence against pathogens ${ }^{18}$. Although a detailed description of these differentially expressed transcripts is not under the main scope of this study that focus on the identification of potential molecular markers useful for mapping, we emphasise those upregulated in response to rust infection only in the partially resistant genotype. This group included transcripts involved in hormone metabolism, cell wall degradation, secondary metabolism, ROS production and in signalling and regulation of

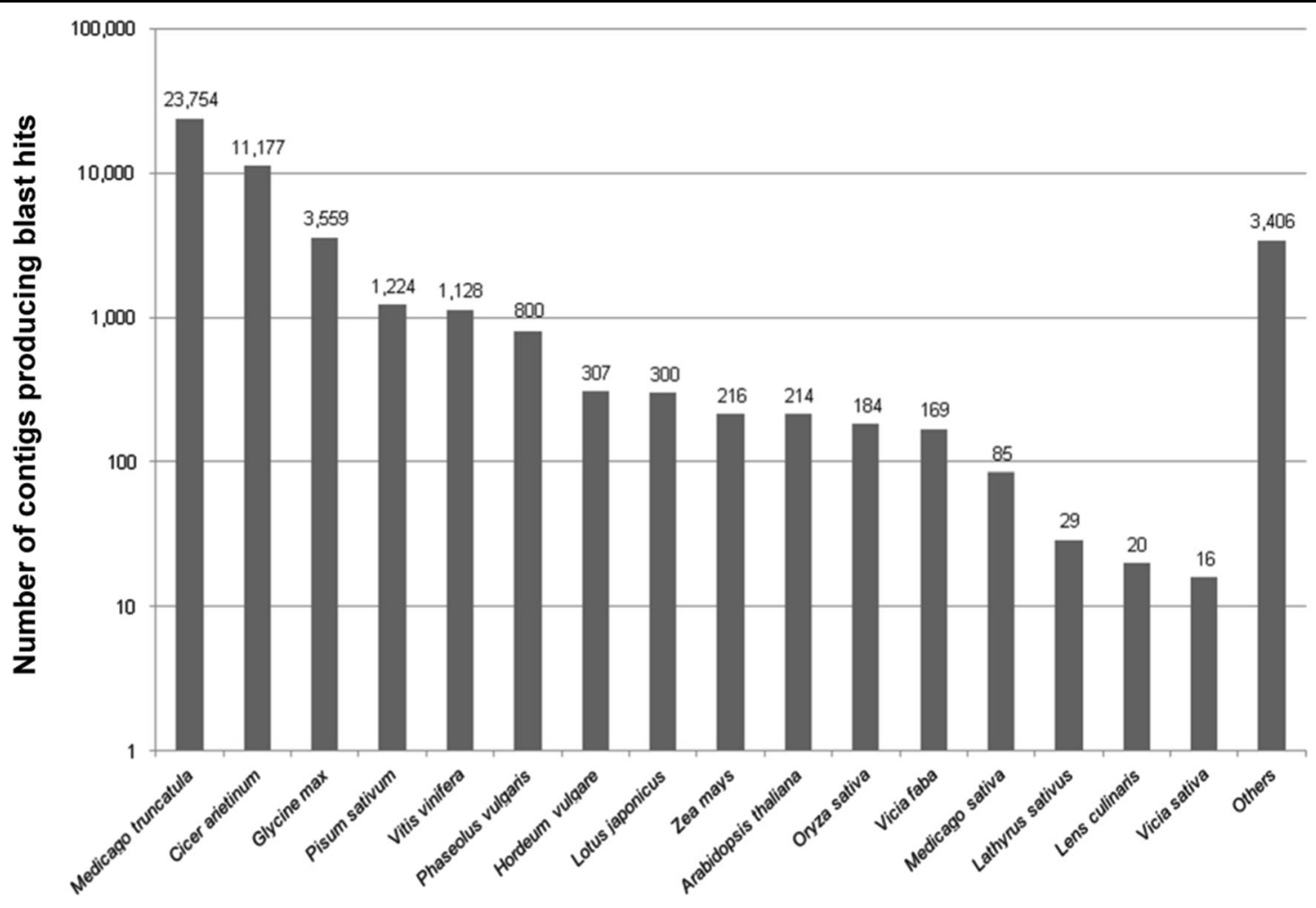

Fig. 1 Number of contigs that could be BLASTed to different plant species 


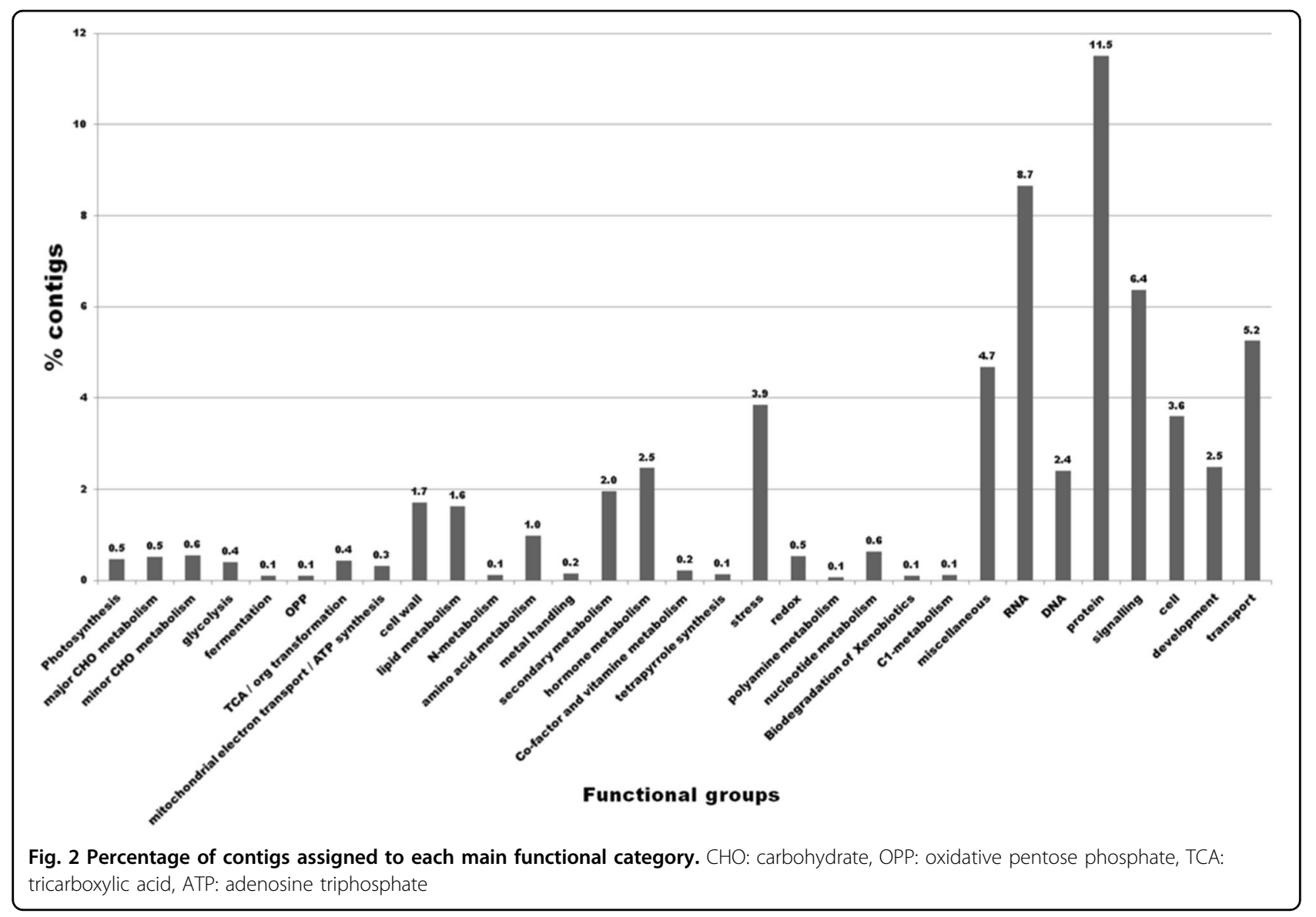

transcription of defence responses. Particularly important for signalling, this group harboured ten receptor kinases, one calcium receptor and a 'WRKY family transcription factor'. Also related to signalling, but in the group of transcripts upregulated in the susceptible and downregulated in the partially resistant genotype, a MLO-like transcript (AtMLO6, PsMLO1, a289255;11) was identified. The complete set of transcripts and its expression profiles can be found in Supplementary Table S1.

\section{RNA-seq validation by qRT-PCR}

To validate the RNA-seq results, the expression of 8 selected genes (chosen to represent a broad range of differential expression and total transcript counts) was analysed by qRT-PCR using three biological replicates. Genes were selected according to their level of differential expression which ranged from $\log _{2}-1.8$ to +2.4 in the two genotypes. Primer sequences are listed in Supplementary Table S2. The normalised number of transcripts was generally higher than 100 , with the exceptions of contig a20510;122, 'Histone H2A.2', with 28 counts in the inoculated BGE008277 and 88 counts in the inoculated BGE023542 sample, and contig a77720;50 ' $\gamma$-tubulin', with 73 counts in the susceptible inoculated line (Supplementary Table S3). The best reference gene assay for normalisation of qRT-PCR reactions for both genotypes, as suggested by the NormFinder software, was the transcript coding for ' $\gamma$-tubulin' (a77720;50). A good correlation $(R=0.71$ for the resistant and $R=0.70$ for the susceptible genotype) was observed between the $\log _{2}$ fold changes measured by RNA-seq and qRT-PCR.

\section{SNP identification in resistance pathways}

A total of 19,224 Single-Nucleotide Polymorphisms (SNPs) were detected in 5,152 of the 43,590 transcripts present in both the susceptible and the partially resistant inoculated genotypes (see Supplementary Fig S1). Among them, 811 SNP-containing contigs had been functionally annotated using the MapMan software. The number of SNPs in functional categories varied considerably. Contigs in the categories 'RNA regulation of transcription' (6.15\%) and 'protein degradation' (4.97\%) contained by far the most SNPs, followed by the proteinrelated categories 'protein postranslational modification' (2.72\%), 'protein synthesis' (1.89\%) and 'protein targeting' $(1.78 \%)$. Also, the categories 'signalling receptor kinases' (1.42\%) and 'hormone metabolism auxin' 
(1.30\%) contained a considerable number of contigs with SNPs (Supplementary Fig. S2). Polymorphic contigs and their respective SNPs are listed in Supplementary Table S4.

Allele-specific expression validation by dual probe assays

Seventeen allele-specific expression assays were tested for their ability to distinguish the two alleles in the genotypes BGE023542 and BGE008277. For eight SNP sites, the allele-specific expression could be confirmed for both alleles (a22544;181, a1871;383, a42821;85, a601;548, a10029;259, a33467;117, a4980;381 and a716;325). In five cases only one of the two alleles could be unambiguously amplified (a16587;204, a12135;196, a28870;97, a1697;460 and a16062;87). For four SNP sites, neither allele could be amplified in both genotypes (a1871;383, a23248;109, a2860;344 and a19261;130) (Table 2).

\section{Development of E-SRR markers}

E-SSRs with a perfect repeat unit of two to six reiterated nucleotides were identified through the Phobos software $^{19}$. In toto we developed assays for 341 expressed SSRs and tested them by PCR amplification of DNA from the two $L$. cicera genotypes. Of these, 251 produced an amplicon, where 207 (60.7\%) amplicons were polymorphic between genotypes BGE008277 and BGE023542, and $44(12.9 \%)$ were monomorphic. Thirty-one $(9.1 \%)$ primer pairs produced a complex pattern and the remaining $59(17.3 \%)$ primer pairs failed to produce any fragment. E-SSR primer pairs and genotyping results are listed in Supplementary Table S5.

\section{Linkage map construction}

A total of 935 molecular markers (767 SNPs, 163 E-SSRs and 5 intron targeted amplified polymorphism (ITAP) markers from a previous study ${ }^{11}$, were screened in the parental genotypes of the F5 RIL population for mapping purposes. From these, 307 polymorphic loci were successfully mapped, namely, 189 SNPs, 113 E-SSRs and 5 ITAPs. In more detail, from the initial 163 E-SSRs screened in the RILs, 126 were selected for mapping, 125 segregated with the expected 1:1 Mendelian ratio (1 degree of freedom; $\left.\alpha=0.01 ; \chi^{2}<3.8\right)$ and one displayed slight segregation distortion $\left(\chi^{2}=4.0\right)$. The remaining markers presented a high segregation distortion and were excluded from further analysis. From the 767 SNPs genotyped in the RIL population, 434 were withdrawn from the mapping due to several causes: 270 had more than $20 \%$ missing values, 162 were monomorphic and 2 were heterozygous in the parental genotypes. A total of 333 SNPs was then selected for developing the linkage map. Of these, 71 segregated severely distorted $(p \leq 0.005)$ and were also removed. From the remaining 262 markers, 66 were removed for having identical segregation to other markers, thus redundant for the map construction. Additionally, one RIL was removed because it had $>25 \%$ of missing data. Finally, the first $L$. cicera linkage map was developed using data from 102 RILs screened with 327 polymorphic loci (126 E-SSRs, 5 ITAPs and 196 SNPs). It covered $724.2 \mathrm{cM}$ of genetic distance organised in 7 major and 2 minor linkages groups (LG), with an average distance of $2.4 \mathrm{cM}$ between markers. Only 5 markers could not be linked to any other LG. Eleven percent of the markers showed significant deviation from the expected 1:1 segregation ratio (segregation distortion), highlighted with asterisks in Fig. 3. A chromosomal region was considered skewed when four or more closely linked markers showed significant segregation distortion in the same direction $^{20}$. In the present linkage map, these were observed in the extremity of LGs V and IX and in the centre of LG I. The smallest LG (IX) mainly contained markers with segregation distortion (Fig. 3).

Inspection of $x^{2}$ values for goodness-of-fit for the individual LGs gave insight into the reliability of the obtained map. The $X^{2}$ values for all the LGs were $<1$, except for LG VIII $\left(x^{2}=1.15\right)$ (Supplementary Table S6).

\section{Macrosynteny between L. cicera LGs and M. truncatula chromosomes}

To obtain insight into the synteny between the $L$. cicera genome and that of other legumes we compared the order of our genic markers to the physical position of $M$. truncatula genome. Clear evidence of a simple and direct macrosynteny between the $L$. cicera and M. truncatula genome was detected as shown in the dot matrix in Fig. 4 and Supplementary Fig. S3. The clear isoclinic diagonal line along the linkage groups in Fig. 4 provided a strong indication of the conservation of gene order in the two legume genomes. However, chromosomal rearrangements were also evident at a moderate level. For example, $M$. truncatula chromosomes 2 and 6 merged to form the $L$. cicera LG II (Fig. 4 and Supplementary Fig. S3, C and G). Similarly, $M$. truncatula chromosome 4 splits into $L$. cicera LGs IV and IX and M. truncatula chromosome 7 into L. cicera LGs VI and VIII (Fig. 4 and Supplementary Fig. S3, E and H, respectively). Additionally, M. truncatula chromosome 8 spans L. cicera LGs VII and a large portion of the distal part of LG IV (Supplementary Fig. S3, I).

\section{Discussion}

Previous studies on pathosystems involving L. cicera described its response to rust $^{11}$, powdery mildew $^{7}$, broomrape $^{9,21}$ and bacterial blight ${ }^{8}$ at the physiological level. The present study contributes to progress in $L$. cicera research and breeding in several respects. First, it provides the sequences of more than 145,985 ESTs representing by far the most extended set of genic sequences available for this species. In addition, it 


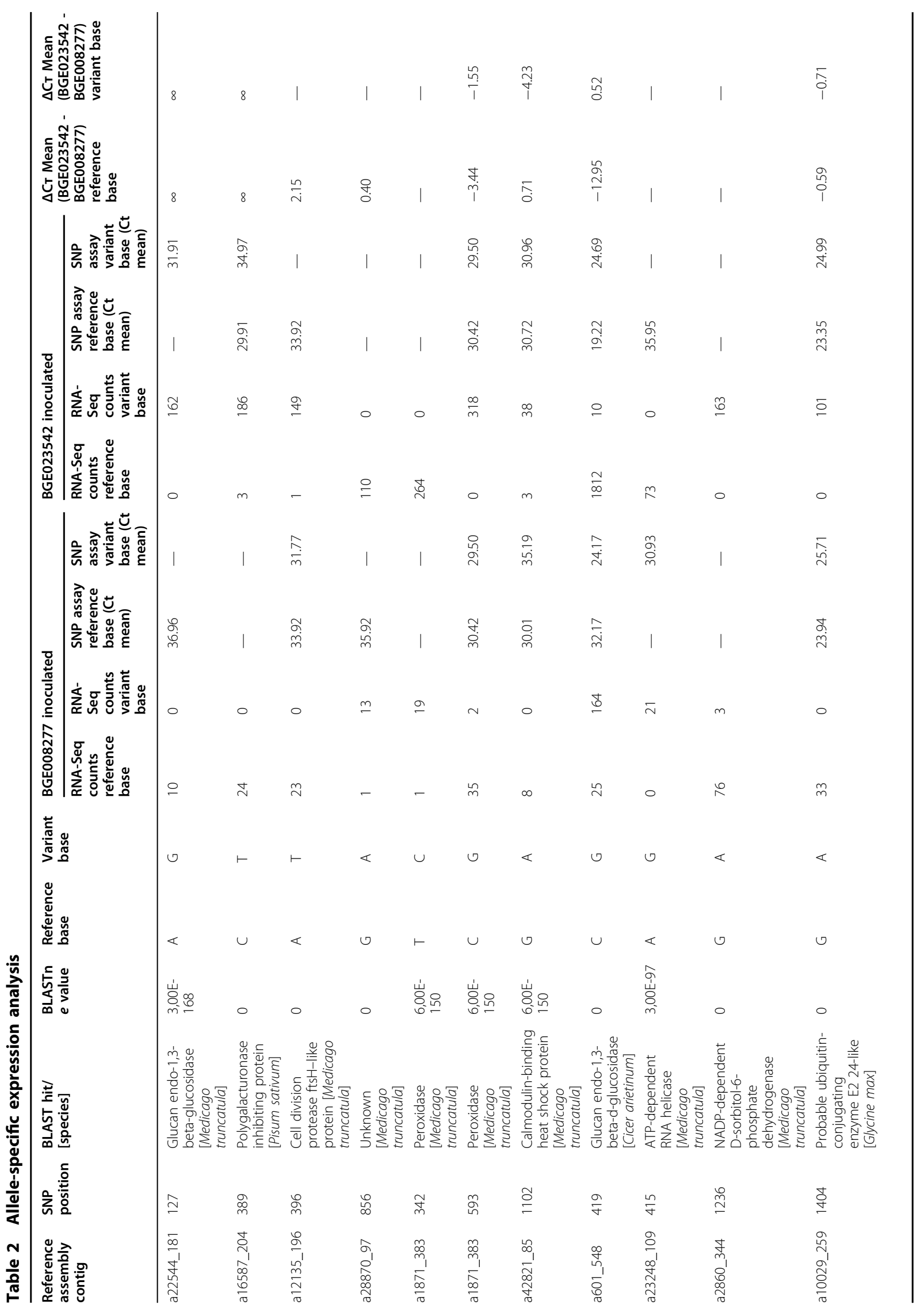




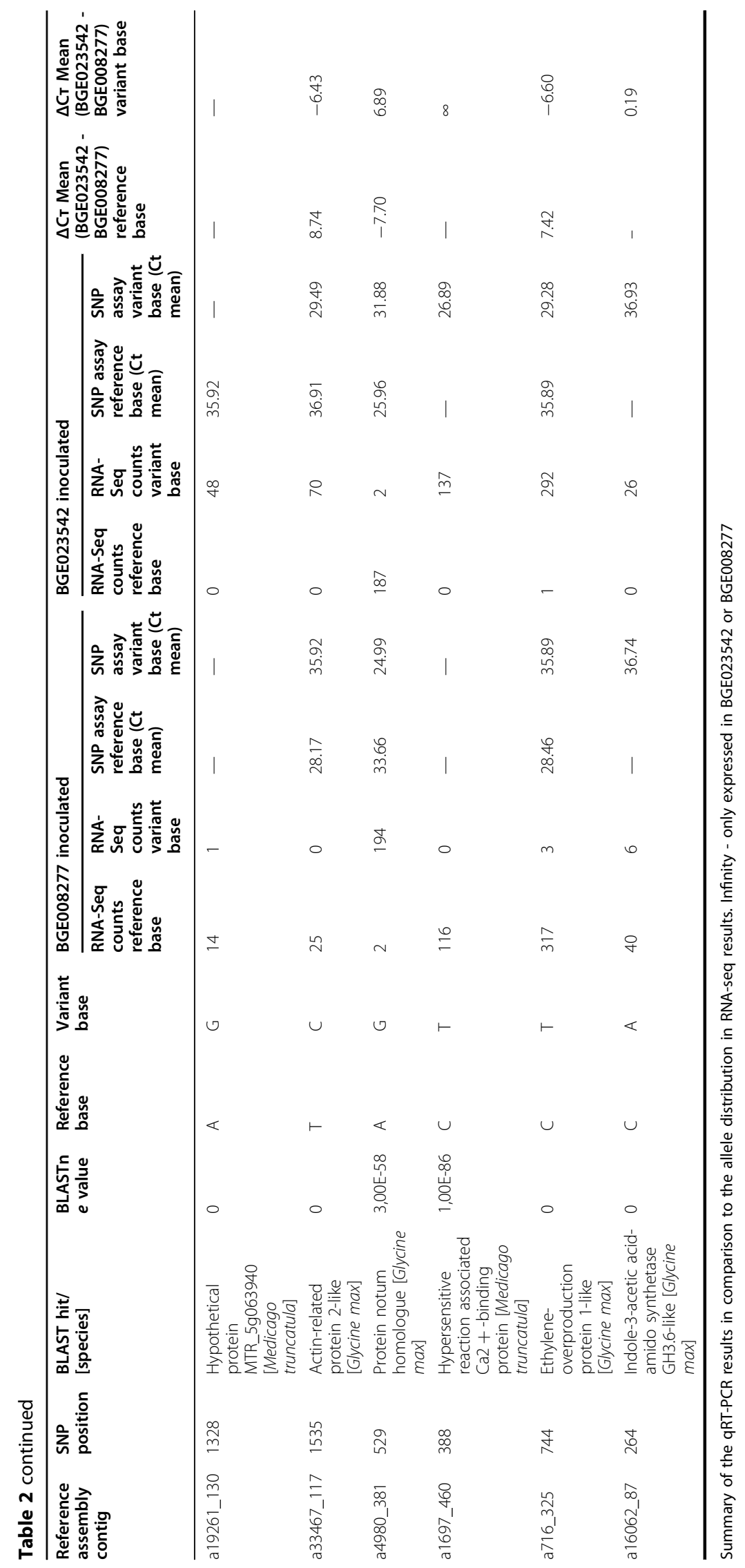




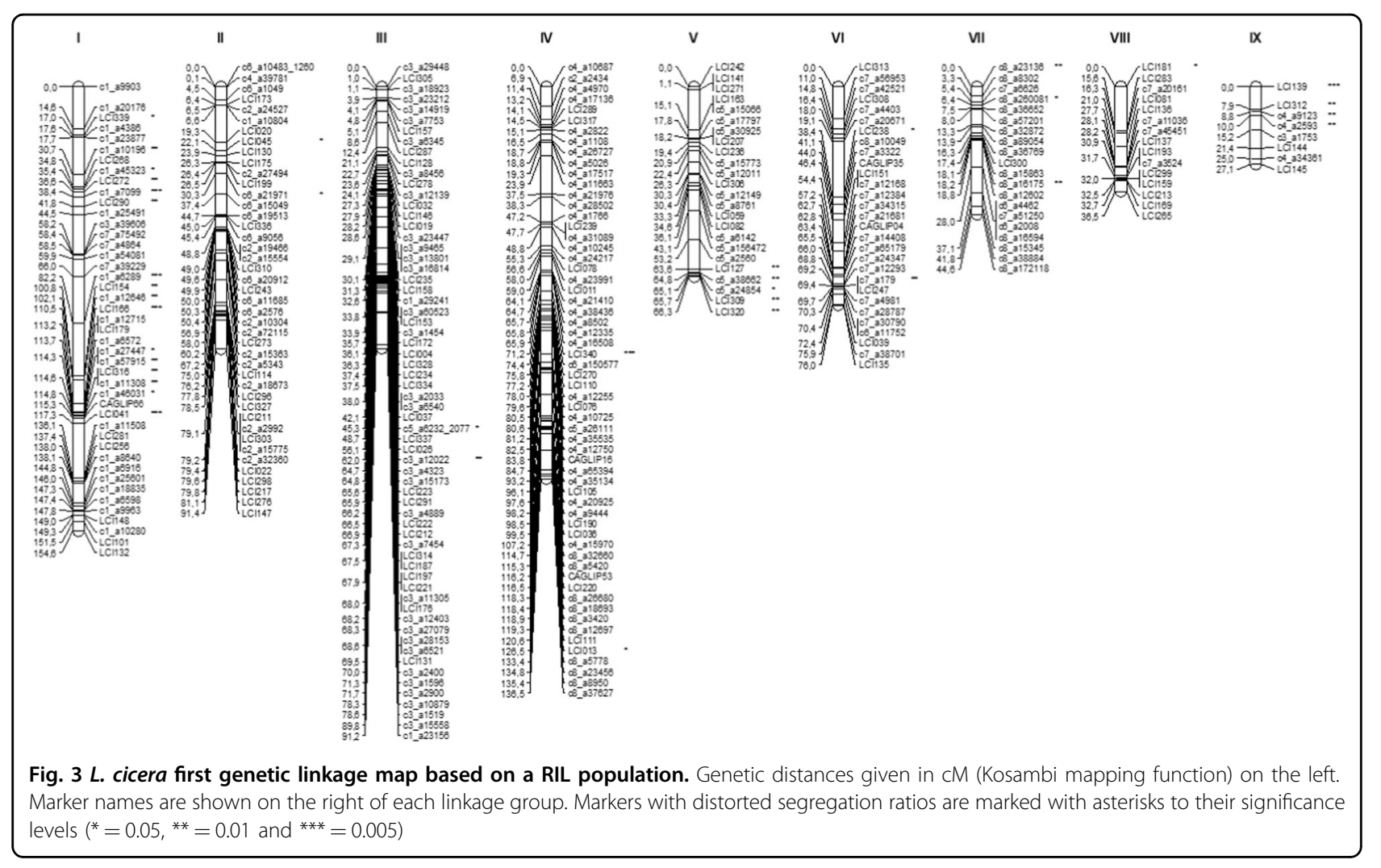

detected 297 potential polymorphic E-SSRs and 19,223 SNPs in more than 5,000 transcripts and tested exemplarily whether these could be converted into molecular markers for later use in molecular breeding and their usefulness in the development of the first $L$. cicera linkage map.

The development of E-SSR markers was quite successful as it resulted in 207 novel, polymorphic marker assays, some of them we employed already here for the construction of a linkage map for $L$. cicera. Eventually these may also be used, by cross-amplification ${ }^{11}$, for genetic mapping in L. sativus. The many other genic SNPs identified here may also be converted into molecular markers. Especially the dual labelled probe qRT-PCR assays that we presented here may become useful for eQTL and allele-specific expression studies. Moreover, additional SNP arrays based on the identified SNP for diversity analysis and genetic mapping in L. cicera can be developed now. This was the case of the Illumina's custom Golden Gate genotyping assay developed by Traitgenetics $\mathrm{GmbH}$, Germany, that we used for genotyping the RILs mapping population in the present study, as described in Material and Methods.

The observed incongruence between the expected and experimental results in the E-SSR validation may be due to the fact that RNA-seq data provide information only from the exons. Therefore, the reason for the failure of several primer pairs to amplify a sequence at all, may be the presence of large introns between the primer binding sites which are not detectable in our RNA-seq data. Also, the production of complex patterns of fragments may be due to the existence of regions of homology to the primer binding sites elsewhere in the genome that were then amplified together with the correct fragment. Eventually, primers were located across splice sites or they could be derived from erroneously assembled contigs ${ }^{22}$. In any case, the fact that $82.4 \%$ of the tested SNPs could be used for the design of functional marker assays holds great promises for further molecular breeding approaches in $L$. cicera. It further demonstrates that the assemblies are correct in most cases.

The fact that only $30 \%$ of our allele-specific expression of candidate resistance gene qRT-PCR assays corroborated the RNA-seq data, points to more complex levels in the regulation of gene expression that need to be further explored $^{23}$. We expect better results from another system with higher resolution. This will eventually increase especially the detection of rare alleles like 'a16062;87' with a maximum of only 40 normalised reads in RNA-seq data (Table 2).

In previous studies, resistance mechanisms against rust appeared to be similar in incompatible $L$. sativus and $L$. cicera genotypes and were consistent with a nonhost resistance response ${ }^{24}$. In both, L. cicera and L. sativus, 


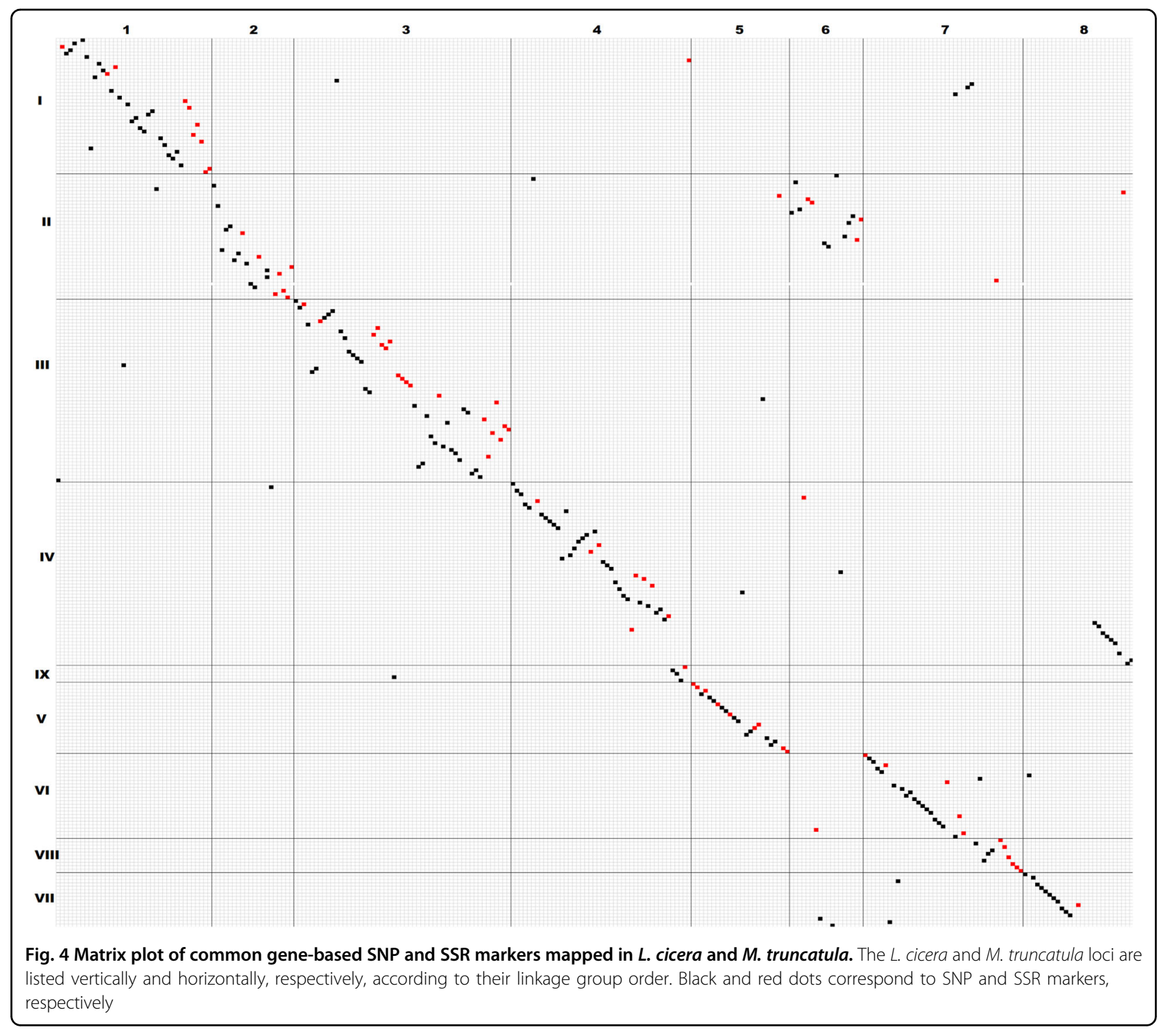

resistant genotypes restrict the formation of haustoria resulting in a high percentage of early aborted fungal colonies, a decreased number of haustoria per colony and reduced intercellular growth of infection hyphae compared to susceptible genotypes from the same species ${ }^{6,11,13}$. Still, L. sativus is generally more resistant than $L$. cicera. For example, the most resistant $L$. cicera genotype (DS $=36 \%$ ) found in an Iberian germplasm collection $^{6}$, is slightly more susceptible than the most susceptible $L$. sativus genotype ( $\mathrm{DS}=30 \%$ ) in the same Iberian Lathyrus germplasm ${ }^{13}$. Future comparative studies should reveal if the same defence-related genes or QTLs are involved in L. cicera and L. sativus resistance to rust, and confirm if the similarities now detected on the phenotypic resistance components would stand at molecular level. As an example, in L. cicera a MLO-like transcript (AtMLO6, PsMLO1, a289255;11) was upregulated in the susceptible and downregulated in the partially resistant genotype (Supplementary Table S1). The MLO gene was first identified in barley, where loss-of-function mutations in this gene conferred resistance to powdery mildew $^{25}$. MLO-like genes mediated the vulnerability to several fungal pathogens in Arabidopsis ${ }^{26}$ and to powdery mildew in Pisum sativum ${ }^{27}$. In our previous study on rust resistance in $L$. sativus, a similar transcript was upregulated in the partially resistant genotype whereas in the resistant genotype this transcript was completely absent ${ }^{17}$. These findings suggest that AtMLO6/PsMLO1 homologs contribute to susceptibility in several Lathyrus spp., a fact that warrants an in-depth exploration in the future.

The lack of genomic resources is a common restraint to molecular analysis in orphan crops, and L. cicera was not an exception. From all the contigs assembled in this study, 
only $42 \%$ were successfully BLASTed to entries in plant databases. Since we sequenced samples inoculated with rust, we could also identify transcripts that probably have a fungal origin. Those were $1.2 \%$ of the total transcripts. As already discussed by Hacquard et al. ${ }^{28}$, the low number of fungal transcripts may reflect the low number of fungal structures in early-infected leaves.

We developed the first linkage map for $L$. cicera based on a RIL population. This linkage map comprises different types of mostly genic molecular markers, including E-SSRs, SNPs and a few ITAPs. A total of 935 molecular markers were screened in 103 individuals from a F5 RIL population segregating for rust response to infection. The obtained map covered a total of $724.2 \mathrm{cM}$, with an average density of one marker every $2.4 \mathrm{cM}$, organised in 9 linkage groups, seven longer than $40 \mathrm{cM}$ and 2 shorter groups. About $11.7 \%$ of mapped markers showed distorted segregation. These were enriched in female parent alleles and were found in clusters mainly in the extremities of linkage groups. Skewed regions may include potential lethal genes $^{29}$, which when homozygous, may produce a lethal phenotype. Therefore, RILs containing those alleles will be absent from the mapping population. Due to linkage drag these genes would then distort the segregation of the whole-genomic region ${ }^{30}$.

Extensive co-linearity was detected between the $L$. cicera linkage groups and the M. truncatula genome. Similarly, high levels of marker order conservation have also been reported between $M$. truncatula and $P$. sati$v u m^{31}$ and other closely related legumes such as $M$. truncatula and M. sativa ${ }^{32}$, L. culinaris and M. truncatula $^{33}$ and $L$. culinaris and $P$. sativum ${ }^{34}$. As in these comparative studies we also identified a few rearrangements in the otherwise syntenic marker order, such as inversions (LG III and IV) and translocations (LG II and IV). In a comparison between $M$. sativa and $P$. sativum syntenic groups ${ }^{35}$, one re-arrangement was similar to the one we observed in L. cicera: linkage group (LG II) from $L$. cicera was split in two chromosomes in $M$. truncatula (Chr. 2 and 6). Also, P. sativum's LG IV contained $M$. sativa's LG 2 and LG 6[35]. We therefore hypothesise that the reduction of the number of chromosomes in $M$. truncatula/M. sativa $(\mathrm{n}=8)$ compared to $L$. cicera/P. sativum $(\mathrm{n}=7)$ is caused by the fusion of $M$. truncatula chromosomes 2 and $6^{36}$. Moreover, a translocation involving chromosomes 4 and 8 of $M$. truncatula that were represented by LG IV in L. japonicus was reported ${ }^{37}$.

In conclusion, our study provides a number of significant genetic resources for L. cicera in particular and in legume genomics in general. For the first time in L. cicera genic SSRs and SNPs were detected and validated for their use as genetic markers. Moreover, the first linkage map for $L$. cicera created with their help can now be used as a tool to localise genes governing desirable traits such as resistance to rust that segregates in the RIL population used to develop the map.

\section{Material and methods Plant material}

The two studied L. cicera genotypes, BGE008277 and BGE023542, have contrasting quantitative resistance to rust infection. Previous evaluation of resistance levels against $U$. pisi demonstrated that BGE008277 is susceptible to rust [disease severity (DS) $=80 \%$ ], whereas BGE023542 is partially resistant (DS $=36 \%)^{6}$. These genotypes were kindly provided by the Plant Genetic Resources Centre (CRF-INIA), Madrid, Spain. The mapping population used for the development of the linkage map consisted of 103 F5 Recombinant Inbred Lines (RILs) derived by single seed descendent from a cross between the two previously described L. cicera genotypes, BGE008277 and BGE023542.

\section{Rust isolate and inoculation}

The $U$. pisi monosporic isolate $\mathrm{UpCo}-01$ from the fungal collection of the Institute for Sustainable Agriculture-CSIC (Córdoba, Spain) was used for inoculation. Fungal spores kept at $-80^{\circ} \mathrm{C}$ were multiplied on plants of the susceptible P. sativum cv. Messire before use.

Twenty-four plants each per genotype (BGE008277/ BGE023542) and treatment (inoculated/control) were used. Two-week-old $L$. cicera seedlings were inoculated by dusting all plants at the same time with $2 \mathrm{mg}$ of spores per plant, diluted in pure talk (1:10), with the help of a small manual dusting device, in a completely randomised experiment. Inoculated and control plants were then incubated for $24 \mathrm{~h}$ at $20^{\circ} \mathrm{C}$ in complete darkness and $100 \%$ relative humidity, then transferred to a growth chamber and kept at $20 \pm 2{ }^{\circ} \mathrm{C}$ under $14 \mathrm{~h}$ light $(150 \mu \mathrm{mol}$ $\mathrm{m}^{-2} \mathrm{~s}^{-1}$ ) and $10 \mathrm{~h}$ dark. Plant response to infection was visually assessed 15 days after inoculation.

\section{RNA and DNA isolation}

Total RNA for RNA-seq, qRT-PCR and SNP validation, was extracted from inoculated and non-inoculated leaves, separately from each individual plant of the two contrasting genotypes. Leaves were collected $37 \mathrm{~h}$ after inoculation, representing a time point in which the fungal colonies are already developing in susceptible Lathyrus genotypes ${ }^{13}$. Collected samples were immediately frozen in liquid nitrogen and stored at $-80^{\circ} \mathrm{C}$. RNA was isolated using the GeneJET Plant RNA Purification Mini Kit (Thermo Scientific, Vilnius, Lithuania) according to the manufacturer's instructions. Isolated RNA was treated with Turbo DNase I (Ambion, Austin, TX, USA), and RNA quantification was carried out using the NanoDrop device (Thermo Scientific, Passau, Germany). For the E-SSR validation and linkage map development, DNA 
from frozen young leaves, from the two contrasting $L$. cicera genotypes and one individual of each RIL, was extracted using a modified CTAB protocol developed by Torres et $a l^{38}$.

\section{RNA sequencing}

For each of the 4 combinations (BGE008277 control and inoculated, BGE023542 control and inoculated) total RNA was extracted individually from 24 plants per genotype and treatment and pooled in equal amounts for sequencing, in order to level out potential outliers. Five RNA-seq libraries (one for each genotype/treatment and one reference assembly which included all genotypes and treatments) were generated using a proprietary protocol (GenXPro GmbH, Frankfurt, Germany). In short, for each library, mRNA was captured from $20 \mu \mathrm{g}$ of total RNA using Oligo dT(25) beads (Dynabeads; life Technologies). The purified mRNA was randomly fragmented in a $\mathrm{Zn}^{2+}$ solution to obtain approximately $250 \mathrm{bp}$ long RNA fragments. cDNA was synthesised by reverse transcription starting from $6(N)$ random hexamer oligonucleotides, followed by second strand synthesis. Barcoded Y-adapters were ligated to the cDNA and the library was amplified with 10 cycles of PCR. The libraries were paired-end sequenced on an Illumina Hiseq2000 machine.

\section{Transcriptome assembly}

Raw sequence reads were passed through quality filtering, thereby also removing sequencing adapters and cDNA synthesis primers. RNA-seq libraries from control and inoculated leaves from each of the two contrasting $L$. cicera genotypes were united prior to assembly to generate a reference assembly, enabling the generation of contigs of maximum length. All high-quality reads were de novo assembled by the Trinity software (Version: trinityrnaseq_r2011-11-26). In order to minimise redundancy, CAP3 software ${ }^{39}$ was also used with overlap length cutoff of $30 \mathrm{bp}$ and overlap percent identity cutoff of $75 \%$. Redundancy was tested using the clustering algorithm UCLUST $^{40}$, available from (http://drive5.com/usearch/ manual/uclust_algo.html). A quality check for assembly completeness was assessed with BUSCO (Benchmarking Universal Single-Copy Orthologs) ${ }^{41}$ against embryophyta_odb9. The resulting contigs were annotated via BLASTX to publicly available plant databases (ftp://ftp. ncbi.nlm.nih.gov/blast/db/FASTA/nr.gz, nr, plants only). To identify potential fungal transcripts, an additional BLASTX to public fungal databases (http://www.ebi.ac.uk/ uniprot, UniProtKB/Swiss-Prot and UniProtKB/TrEMBL) was performed.

The sequenced reads were quantified by mapping to our own de novo assembled contigs with novoalign (V2.07.14; http://www.novocraft.com/). RPKM (reads per kilobase per million) were calculated as the normalised transcript expression value ${ }^{42}$. The obtained counts were subsequently passed through DEGSeq to calculate differential gene expression ( $\mathrm{R}$ package version 1.16.0) ${ }^{43}$.

\section{Contig annotation and data analysis}

In order to classify the obtained contigs into functional categories, the Mercator pipeline for automated sequence annotation $^{44}$, available at http://mapman.gabipd.org/web/ guest/app/mercator, was used. The mapping file was created with information from the following manually curated databases: Arabidopsis TAIR proteins (release 10), SwissProt/UniProt Plant Proteins (PPAP), TIGR5 rice proteins (ORYZA), Clusters of orthologous eukaryotic genes database (KOG), Conserved domain database (CDD) and InterPro scan (IPR). The Mercator mapping file was then employed for pathway analysis by the MapMan software ${ }^{18}$, available at http://mapman.gabipd. org/web/guest/mapman.

Differentially expressed contigs were identified and categorised in expression pattern groups by comparing their expression in leaves of the partially resistant genotype BGE023542, control vs. inoculated, and of the susceptible genotype BGE008277, control vs. inoculated, using DEGseq ${ }^{43}$.

\section{RNA-seq validation by quantitative RT-PCR assay}

To validate the RNA-seq results, expression levels of 8 selected genes were analysed by qRT-PCR. These genes were chosen to represent a broad range of differential expression and total transcript counts. $1 \mu \mathrm{g}$ of total RNA from each of three randomly chosen plants (3 biological replicates), from the twenty-four plants used for the RNA-seq experiment pool, per genotype per treatment (inoculated/control), was reverse transcribed in technical duplicates, using the High Capacity cDNA Reverse Transcription Kit (Applied Biosystems, Foster City, CA, USA) following the manufacturer's instructions, in a total of six samples per genotype and per treatment. qRT-PCR reactions were performed with an $\mathrm{iQ}^{\mathrm{m} m} 5$ Real-Time PCR Detection System (Bio-Rad, Munich, Germany). Primers were designed using the Primer3 software ${ }^{45}$. Primer sequences are listed in Supplementary Table S2. For differential expression data analysis, the Genex software package (MultiD, Goteborg, Sweden) was employed. The NormFinder software ${ }^{46}$, also included in Genex, was used to select the reference gene, considering the expression of $\gamma$-tubulin (a77720;50), Histone H2A.2 (a20510;122) and P700 Phospholipase (a160;902).

\section{SNP detection}

SNPs distinguishing the transcripts of the two inoculated L. cicera genotypes were discovered by JointSNVMix ${ }^{47}$, taking as input the mappings (bam files) from the transcriptome analysis (see section "RNA sequencing" 
and "Transcriptome assembly"). As reference, the contigs used were obtained according to the procedure described in section "Transcriptome assembly". The output of the JointSNVMix analysis was furthermore processed by GenXPro's in-house software to detect SNPs discriminating the variant alleles in the inoculated samples. A minimum coverage of 15 reads in each genotype and a probability of joint genotype BB_AA and AA_BB bigger than 0.98 was needed to call a SNP.

\section{Allele-specific expression analysis by dual labelled probe qRT-PCR assays}

In order to analyse the differential expression of allelic variants, SNPs in genes of interest discriminating the genotypes BGE023542 and BGE008277 were selected, taking into account their expression patterns, for the design of allele-specific qRT-PCR assays (Supplementary Table S7). These comprised 11 transcripts that were equally expressed between the genotypes (a11871;383 transcript present two SNP sites), one transcript with higher expression in the partially resistant genotype (a22544;181) and four transcripts with higher expression in the susceptible genotype (a4980;381, a1697;460, a716;325 and a16062;87). Altogether $2 \times 17$ allele-specific dual labelled probe qRT-PCR assays, with SNP-specific mismatch primers, were tested.

The primers for the dual labelled probe qRT-PCR assays (Supplementary Table S7) were designed with introduced additional mismatch in the forward or reverse primer to improve allele-specific amplification ${ }^{48,49}$. The expected amplicons had a size of $<90 \mathrm{bp}$ in order to avoid background by flanking additional SNPs (see documentation of the primer design in the Supplementary Table S8). The one-step qRT-PCR reactions were performed with 40-60 ng total RNA template per reaction from BGE023542 and BGE008277 and the corresponding 5'6-Fam-3'TQ2 dual labelled probe and the different mismatch primer for the SNP alleles in separate tubes. All reactions were made in $12 \mu \mathrm{l}$ of One Step Prime Script ${ }^{\mathrm{max}}$ RT-PCR Kit buffer (Perfect Real Time) from TAKARA Bio Inc., Japan. The qRT-PCR regime consisted of a reverse transcription step of $5 \mathrm{~min}$ at $42^{\circ} \mathrm{C}$ and an initial denaturation step of $10 \mathrm{~s}$ at $95^{\circ} \mathrm{C}$, followed by 40 cycles of $5 \mathrm{~s}$ at $95^{\circ} \mathrm{C}$ (denaturation) and $30 \mathrm{~s}$ at $62^{\circ} \mathrm{C}$ (annealing/ elongation).

\section{Development of E-SSR}

The de novo transcriptomes were in silico searched for perfect SSRs with a repeat unit length of two to six nucleotides employing the Phobos ${ }^{19}$ plug-in for Geneious software ${ }^{50}$. Length polymorphisms were manually identified by aligning SSR-containing contigs of one genotype against the whole library of the other genotype.

\section{Molecular markers screening and map construction}

The DNA from each RIL individual in addition to the two parental lines was screened using a subset of the previously developed molecular markers. In this way, 163 E-SSR markers predicted in silico from L. cicera parental genotypes RNA-Seq libraries plus 767 SNPs, selected from the same libraries taking into consideration their homology with the Medicago truncatula genomic sequence (MT3.5) (BLASTn; $E$ value $<1 \mathrm{E}-6)$ and their physical position in this genome to cover evenly M. truncatula's chromosomic regions (http://www. medicagohapmap.org/tools/blastsearch), preventing unwanted clustering of markers, were screened. In addition, and for the linkage map construction also five heterologous ITAP markers selected from previous publications ${ }^{11}$, identified as polymorphic and with an 1:1 Mendelian segregation in this mapping population were used. PCR reactions and genotyping were performed as described in Almeida et al. ${ }^{17}$, with the exception of SNP markers that were genotyped using an Illumina's custom Golden Gate genotyping assay provided by Traitgenetics $\mathrm{GmbH}$, Germany.

Linkage analysis and segregation distortion tests were performed using JoinMap 4.0 software $^{54}$, using a binary matrix including all the genotyping data as input. Markers with a severe segregation distortion $(p \leq 0.005)$ were removed from the original molecular data set. The determination of groups of linked markers (linkage groups-LGs) was done with a LOD score of 4. Linkage map calculations were done using all pairwise recombination estimates lower than 0.40 and a LOD score higher than 1.00, applying the Kosambi mapping function ${ }^{51}$. The reliability of the obtained map was checked by inspecting the individual LG $\chi^{2}$ value.

\section{Comparison with $M$. truncatula genome}

Using the order of the genic SNP and SSR markers in the $L$. cicera linkage map and the information of the physical position of the same markers mapped on the M. truncatula genomic sequence (MT4.0) $)^{52}$ (BLASTn; $E$ value $<1 \mathrm{E}-6)$, markers were aligned in a matrix. Co-linearity was also investigated using Strudel visualisation software ${ }^{53}$. Columns from the matrix correspond to the M. truncatula genomic sequence and rows correspond to the L. cicera LGs rearranged in order to facilitate the visual estimation of co-linearity.

\section{Acknowledgements}

We thank the CRF-INIA, Madrid, Spain, for supplying the genotypes. This work was supported by the project LEGATO (FP7 grant agreement $n^{\circ}$ 613551), the Spanish grant AGL2017-82907-R and the Portuguese Grants PTDC/AGR-TEC/ 0992/2014 and UID/Multi/04551/2013 by Fundação para a Ciência e

Tecnologia. N.F.A. and M.C.V.P. were supported by Fundação para a Ciência e Tecnologia (SFRH/BD/44357/2008 and IF/01337/2014 Research Contract by IF 2014 program, respectively). We also acknowledge COST Action Sustain 
(FA1208 - Pathogen-informed strategies for sustainable broad-spectrum crop resistance).

\section{Author details}

${ }^{1}$ Instituto de Tecnologia Química e Biológica António Xavier, Universidade Nova de Lisboa, Av. da República, Oeiras 2780-157, Portugal. GenXPro GmbH, Frankfurt am Main D-60438, Germany. ${ }^{3}$ Institute for Sustainable Agriculture, CSIC, Córdoba E-14004, Spain

\section{Author Contributions}

N.F.A. carried out the inoculations and sample processing for sequencing and qRT-PCR, designed the GRT-PCR primers, and developed the E-SSRs and SNP primers. C.S. and M.L.A. participated in the construction of the linkage map. C.S. and N.F.A. performed the comparative mapping and drafted de manuscript. R. H. designed and performed the allele-specific dual probe assays. N.F.A. and S.T. $L$. participated in the results generation on qRT-PCR and respective data analysis. C.S., S.T.L. and T.A-F. performed the E-SSR genotyping. N.K. and B.R. performed the RNA sequencing and bioinformatics data processing. D.R. developed the RIL population. P.W. and D.R. revised the manuscript critically. M.C.V.P. coordinated the study and participated in the drafting and revision of the manuscript.

\section{Conflict of interest}

The authors declare no conflict of interest.

Supplementary Information accompanies this paper at https://doi.org/ 10.1038/s41438-018-0047-9.

Received: 11 January 2018 Revised: 5 March 2018 Accepted: 30 April 2018 Published online: 01 September 2018

\section{References}

1. Kenicer, G. J., Kajita, T., Pennington, R. T. \& Murata, J. Systematics and biogeography of Lathyrus (Leguminosae) based on internal transcribed spacer and cpDNA sequence data. Am. J. Bot. 92, 1199-1209 (2005).

2. Schaefer, H. et al. Systematics, biogeography, and character evolution of the legume tribe Fabeae with special focus on the middle-Atlantic island lineages. BMC Evol. Biol. 12, 250 (2012).

3. Hanbury, C. D., Siddique, K. H. M., Galwey, N. W. \& Cocks, P. S. Genotypeenvironment interaction for seed yield and ODAP concentration of Lathyrus sativus L. and L. cicera L. in Mediterranean-type environments. Euphytica 110 , 45-60 (1999).

4. Peña-Chocarro, L. \& Peña, L. Z. History and traditional cultivation of Lathyrus sativus L. and Lathyrus cicera L. in the Iberian peninsula. Veg. Hist. Archaeobot. 8, 49-52 (1999).

5. Silvestre, S., de Sousa Araújo, S., Vaz Patto, M. C. \& Marques da Silva, J. Performance index: an expeditious tool to screen for improved drought resistance in the Lathyrus genus. J. Integr. Plant Biol. 56, 610-621 (2014).

6. Vaz Patto, M. C., Fernández-Aparicio, M., Moral, A. \& Rubiales, D. Pre and posthaustorial resistance to rusts in Lathyrus cicera L. Euphytica 165, 27-34 (2009).

7. Vaz Patto, M. C., Fernández-Aparicio, M., Moral, A. \& Rubiales, D. Resistance reaction to powdery mildew (Erysiphe pisi) in a germplasm collection of Lathyrus cicera from Iberian origin. Genet. Resour. Crop Evol. 54, 1517-1521 (2007).

8. Martín-Sanz, A., Pérez de la Vega, M. \& Caminero, C. Resistance to Pseudomonas syringae in a collection of pea germplasm under field and controlled conditions. Plant Pathol. 61, 375-387 (2012).

9. Fernández-Aparicio, M., Flores, F. \& Rubiales, D. Field response of Lathyrus cicera germplasm to crenate broomrape (Orobanche crenata). Field Crops Res. 113, 321-327 (2009).

10. Vaz Patto, M. C. \& Rubiales, D. Lathyrus diversity: Available resources with relevance to crop improvement - L. sativus and L. cicera as case studies. Ann. Bot. 113, 895-908 (2014).

11. Almeida, N. F. et al. Transferability of molecular markers from major legumes to Lathyrus spp. for their application in mapping and diversity studies. Mol. Biol. Rep. 41, 269-283 (2014).
12. Barilli, E., Sillero, J. C., Serrano, A. \& Rubiales, D. Differential response of pea (Pisum sativum) to rusts incited by Uromyces viciae-fabae and U. pisi. Crop Prot. 28, 980-986 (2009).

13. Vaz Patto, M. C. \& Rubiales, D. Identification and characterization of partial resistance to rust in a germplasm collection of Lathyrus sativus L. Plant Breed. 128, 495-500 (2009).

14. Barilli, E., Moral, A., Sillero, J. C. \& Rubiales, D. Clarification on rust species potentially infecting pea (Pisum sativum L.) crop and host range of Uromyces pisi (Pers.) Wint. Crop Prot. 37, 65-70 (2012).

15. Rubiales, D., Sillero, J. C. \& Emeran, A. A. Response of vetches (Vicia spp.) to specialized forms of Uromyces vicia-fabae and to Uromyces pisi. Crop Prot. 46, 38-43 (2013).

16. Hopf, M. in The Origin and domestication of cultivated plants, Vol. 16 (ed Barigozzi, C.) Pages 35-60 (Elsevier Science, 1986).

17. Almeida, N. F. et al. Allelic diversity in the transcriptomes of contrasting rustinfected genotypes of Lathyrus sativus, a lasting resource for smart breeding. BMC Plant Biol. 14, 376 (2014).

18. Thimm, O. et al. MAPMAN: A user-driven tool to display genomics data sets onto diagrams of metabolic pathways and other biological processes. Plant $\mathrm{J}$. 37, 914-939 (2004)

19. Mayer, C. Phobos 3.3.11. http://www.rub.de/ecoevo/cm/cm_phobos.htm (2010).

20. Xu, Y., Zhu, L., Xiao, J., Huang, N. \& McCouch, S. R. Chromosomal regions associated with segregation distortion of molecular markers in F2, backcross, doubled haploid, and recombinant inbred populations in rice (Oryza sativa L.). Mol. Gen. Genet. 253, 535-545 (1997).

21. Fernández-Aparicio, M. \& Rubiales, D. Characterisation of resistance to crenate broomrape (Orobanche crenata Forsk.) in Lathyrus cicera L. Euphytica 173, 77-84 (2010).

22. Varshney, R. K., Graner, A. \& Sorrells, M. E. Genic microsatellite markers in plants: features and applications. Trends Biotechnol. 23, 48-55 (2005).

23. Zhang, X. \& Borevitz, J. O. Global analysis of allele-specific expression in Arabidopsis thaliana. Genetics 182, 943-954 (2009).

24. Vaz Patto, M. C. \& Rubiales, D. Unveiling common responses of Medicago truncatula to appropriate and inappropriate rust species. Front. Plant Sci. 5, 618 (2014).

25. Jørgensen, I. H. Discovery, characterization and exploitation of Mlo powdery mildew resistance in barley. Euphytica 63, 141-152 (1992).

26. Chen, Z. et al. Expression analysis of the AtMLO gene family encoding plantspecific seven-transmembrane domain proteins. Plant Mol. Biol. 60, 583-597 (2006).

27. Humphry, M., Reinstädler, A., Ivanov, S., Bisseling, T. \& Panstruga, R. Durable broad-spectrum powdery mildew resistance in pea er 1 plants is conferred by natural loss-of-function mutations in PSMLO1. Mol. Plant Pathol. 12, 866-878 (2011).

28. Hacquard, S. et al. The poplar-poplar rust interaction: insights from genomics and transcriptomics. J. Pathog. 2011, 1-11 (2011).

29. Cheng, R., Kleinhofs, A. \& Ukai, Y. Method for mapping a partial lethal-factor locus on a molecular-marker linkage map of a backcross and doubled-haploid population. Theor. Appl. Genet. 97, 293-298 (1998).

30. Törjék, O. et al. Segregation distortion in Arabidopsis C24/Col-0 and Col-0/C24 recombinant inbred line populations is due to reduced fertility caused by epistatic interaction of two loci. Theor. Appl. Genet. 113, 1551-1561 (2006).

31. Aubert, G. et al. Functional mapping in pea, as an aid to the candidate gene selection and for investigating synteny with the model legume Medicago truncatula. Theor. Appl. Genet. 112, 1024-1041 (2006).

32. Choi, H. K. et al. A sequence-based genetic map of Medicago truncatula and comparison of marker colinearity with M. sativa. Genetics 166, 1463-1502 (2004).

33. Phan, H. T. T. et al. Extensive macrosynteny between Medicago truncatula and Lens culinaris ssp. culinaris. Theor. Appl. Genet. 114, 549-558 (2007).

34. Weeden, N. F., Muehlbauer, F. J. \& Ladizinski, G. Extensive consenvation of linkage relationships between pea and lentil genetic maps. J. Hered. 83, 123-129 (1992)

35. Kaló, P. et al. Comparative mapping between Medicago sativa and Pisum sativum. Mol. Genet. Genom. 272, 235-46 (2004).

36. Choi, H.-K. et al. Estimating genome conservation between crop and model legume species. Proc. Natl Acad. Sci. 101, 15289-15294 (2004).

37. Young, N. D. et al. Sequencing the genespaces of Medicago truncatula and Lotus japonicus. Plant Physiol. 137, 1174-1181 (2005). 
38. Torres, A. M., Weeden, N. F. \& Martín, A. Linkage among isozyme, RFLP and RAPD markers in Vicia faba. Theor. Appl. Genet. 85, 937-945 (1993).

39. Huang, X. \& Madan, A. CAP3: A DNA sequence assembly program. Genome Res. 9, 868-877 (1999).

40. Edgar, R. C. Search and clustering orders of magnitude faster than BLAST. Bioinformatics 26, 2460-2461 (2010).

41. Waterhouse, R. M. et al. BUSCO applications from quality assessments to gene prediction and phylogenomics. Mol. Biol. Evol. 35, 543-548 (2017).

42. Marioni, J. C., Mason, C. E., Mane, S. M., Stephens, M. \& Gilad, Y. RNA-seq: an assessment of technical reproducibility and comparison with gene expression arrays. Genome Res. 18, 1509-1517 (2008).

43. Wang, L., Feng, Z., Wang, X., Wang, X. \& Zhang, X. DEGseq: an R package for identifying differentially expressed genes from RNA-seq data. Bioinformatics 26, 136-138 (2009).

44. Lohse, M. et al. Mercator: a fast and simple web server for genome scale functional annotation of plant sequence data. Plant, Cell Environ. 37, 1250-1258 (2014)

45. Untergasser, A. et al. Primer3-new capabilities and interfaces. Nucleic Acids Res. 40, e115 (2012).

46. Andersen, C. L., Jensen, J. L. \& Ørntoft, T. F. Normalization of real-time quantitative reverse transcription-\{PCR\} data: a model-based variance estimation approach to identify genes suited for normalization, applied to bladder and colon cancer data sets TL - 64. Cancer Res 64 VN-r, 5245-5250 (2004).

47. Roth, A et al. JointSNVMix: a probabilistic model for accurate detection of somatic mutations in normal/tumour paired next-generation sequencing data. Bioinformatics 28, 907-913 (2012).

48. Kwok, S., Chang, S. Y., Sninsky, J. J. \& Wang, A. A guide to the design and use of mismatched and degenerate primers. Genome Res. 3, S39-47 (1994).

49. Liu, J. et al. An improved allele-specific PCR primer design method for SNP marker analysis and its application. Plant Methods 8, 34 (2012).

50. Drummond, A. J. et al. Geneious v5.4 http://www.geneious.com (2011).

51. Kosambi, D. D. The geometric method in mathematical statistics. Am. Math Mon. 51, 382 (1944).

52. Tang, $\mathrm{H}$. et al. An improved genome release (version Mt4.0) for the model legume Medicago truncatula. BMC Genom. 15, 312 (2014).

53. Bayer, M. et al. Comparative visualization of genetic and physical maps with strudel. Bioinformatics 27, 1307-1308 (2011).

54. Van Ooijen, J. W. JoinMap 4, Software for the calculation of genetic linkage maps in experimental populations. Kyazma B. V., Wageningen, Netherlands (2006). 